\title{
Parking Slot Reservation System for Efficient Resource Utilization and Profit
}

\author{
Kanaga Suba Raja.S ${ }^{a}$, Usha Kiruthika ${ }^{b}$, R. Jaichandran ${ }^{c}$, Kamala Kannan .R ${ }^{d}$, Kiran Kumar .A ${ }^{e}$ and \\ Aditya .R $\mathbf{R}^{\mathbf{f}}$
}

A

Easwari Engineering College, Chennai -600 089.

bSR Institute of Science and Technology, Kattankulathur - 603203

${ }^{\mathbf{c}}$ Aarupadaiveedu Institute of Technology, VinayakaMission's Research Foundation,

Chennai

dEaswari Engineering College, Chennai -600 089

${ }^{\mathrm{e} E}$ Easwari Engineering College, Chennai -600 089

fEaswari Engineering College, Chennai -600 089

Article History: Received: 11 January 2021; Accepted: 27 February 2021; Published online: 5 April 2021

\begin{abstract}
Theparkingspacesavailableindaily moving lifestyle is not considered for parking. This problem causes many people to park their vehicles road side and results traffic violations and many accidents in major cities. These can be avoided when people can find the available parking spaces and reserve them to park their vehicles before reaching to that specific area. The main idea is based on mobile applications where the client and the vendor uses mobile applications for their purposes. The client can viewavailableparkingareasintheirdestination and can book them in advance before reaching them. The vendor application can be used by the parking lot owners to authenticate the incoming vehicles and allocate slots for both the client booked with application and naive users coming without using client application. The main algorithms used provides profit to both the users and the parking lot owners and best slot allocation algorithm. By this way many unused and distance parking lots can be utilisedinordertoreducetheroadsideparkings.
\end{abstract}

Keywords: parking, real-time, reservation, payment, estimation, sensors, management, client application

\section{Introduction}

Parking is a very major problem in day to day life of a person. Given the poorly managed and maintained system of parking, the efforts put into finding a proper and safe parking space is onlyincreasingexponentially.InIndia,wehave parking spaces all over the city, but we don't haveasysteminplacethatletsthepeopleknow about the whereabouts of an available parking space. This poorly managed,infrastructure-less parking method increases the time and fuel consumption of the daily public causing frustration. The pain point in this existing system is that the public don't have any knowledge about the availability of a parking space in peak hours.Developing a system that gives the users all the required information and letting them reserve these parking space, reduces the traffic congestion caused due to parking inconvenience and also the time and fuel consumption.

\section{Literature Survey}

"A Cloud-Based Smart-Parking System Based on Internet-of-Things Technologies" in 2015 Uses a novel algorithm that increases the efficiency of the current cloud-based smart- parking system and develops a network architecture based on the Internet-of-Things technology Find the

Available parking space Use cost-based metric tofindthecheapparkingspaceCannotestimate the parking time Does not have a dedicated monitoring system inplace. In 2017, "An IoT-based E-Parking System for Smart Cities" Uses an integrated component called parking meter to address the parking issues as well as to provide smart parking management throughout the city Uses a mobile applicationtobooklotsMakepaymenteasyvia theapplicationNotscalableforimplementation High cost ofimplementation

In 2018, "An Android Application for Smart Parking with Efficient Space Management"

Android application for smart parking with efficient space management which offers full automation of paying for parking. Making paymenteasyforthepublicwhentheyareusing an open parking space Only addresses the payment perspective Does not allow users to book parkinglots.

"Smart parking sensors, technologies and applications for open parking lots: a review" in 2018[11][12]. This study reviews the literature on the usage of smart parking sensors, technologies, applications and evaluates their applicability to open parking lots. Studies the use of various sensors in parking assistance. This is just a review on the parking system. Does not have any real implementation. 


\section{PROPOSED SYSTEM}

The proposed system consists of IR monitored parking space and a client application at the disposal of the user. The parking has multiple parking lots that are individually monitored using IR sensors and connected through a sensor interface, which is then connected tothe centralserver.

The other integral part of this proposedsystem is the estimation algorithm. This

algorithmwillestimatetheparkingtimeofeach user. The server will use this estimated time as a parameter and update the availability of the parkinglots.

This estimation algorithm increases the efficiency of the system by allowing people toreserve parking very well in advance.

The client application is used by the public to search for free parking at their desired destination.Userscancheckfortheavailability oflotsinthoseparkingspacesandreservethem for their use when they reach theirdestination.

The user has to register using their license number and also their vehicle's registration number. This provides the system with enough integrity and security.

Once registered, the user can search for available parking spaces in their desired destination and select a parking space. Then they can check for the number of available parking lots from which they can select one for their use. After selecting they can confirmtheir reservation, after which they will receive a confirmationmessage.

On reaching their destination, the user can activate their parking lot by scanning a unique QR code that has been generated in the client application.AfterscanningtheQRcode, allthe details of the user are verified and the lot is activated.

The IR sensors start monitoring the lot after they get activated. The user can complete the parking by using the client application. The status of the parking is cross-verified using the IR sensors.

Once the parking has been completed and verified, a QR code is generated in the client application once again to authenticate the user. This code is scanned and then a bill is generated. The user can pay the bill using any payment e-wallet or net banking options.

\section{IMPLEMENTATION}

Algorithm Used

INPUT: Destination and Time

OUTPUT: ParkingAvailability

1. Construct a training dataset containing the destination and averageparkingtime.Anestimation algorithmisbasedonaclassification structuredevelopedusingamachine learningalgorithm.

2. Read the destination as the input from the user and also the time for reservation.

3. StorethedestinationinavariableX, the location of the destination is extracted using the Google Maps API. Based on the location, the destination details arefetched.

4. The destination is then fed into the classification algorithm that classifies thedestination/.

5. Based on the class label, the estimation algorithm estimates the average parking time of eachlot.

6. Comparingtheaverageparkingtime and the input reservation time, the server returns the availability of the slot.

a. ExpectedOutcomes

Input: Phoenix Market City, 10AM

Processing:

Phoenix Market City - Co-ordinates are extracted.

Classification:

Based on the co-ordinates, the destination details are fetched.

The location is classified as a Mall basedon the destinationdetails

Time comparison:

Based on the class label - Mall, the algorithm calculates the average parking time.

Average parking time - avg_time

Input reservation parking time - park_time If(avg_time<park_time)

Slot_availability = yes;

Else

Slot_availability $=$ no;

b. Modules:

User Authentication Module

- Read user_name,password 
- Check if user_name isvalid,

o Ifinvalid,

Ask the user to register for a newaccount

o Ifvalid,

Retrieve the password for the particularuser

Check if password entered is the rightone

- If password isvalid,

o Redirect the user to the next screen.

- If password isinvalid,

o Ask the user to enter the right credentials.

Vehicle AuthenticationModule

- Readlicense_plate_no

- User captures photo of licenseplate

- Extract license plate no frompicture

- Compare both thenumbers

- Ifsame,

o Register the vehicle and store thedata

- If notsame,

o Ask the user to re-enter the license_plate_no

o Recapture the licenseplate

o Compareagain

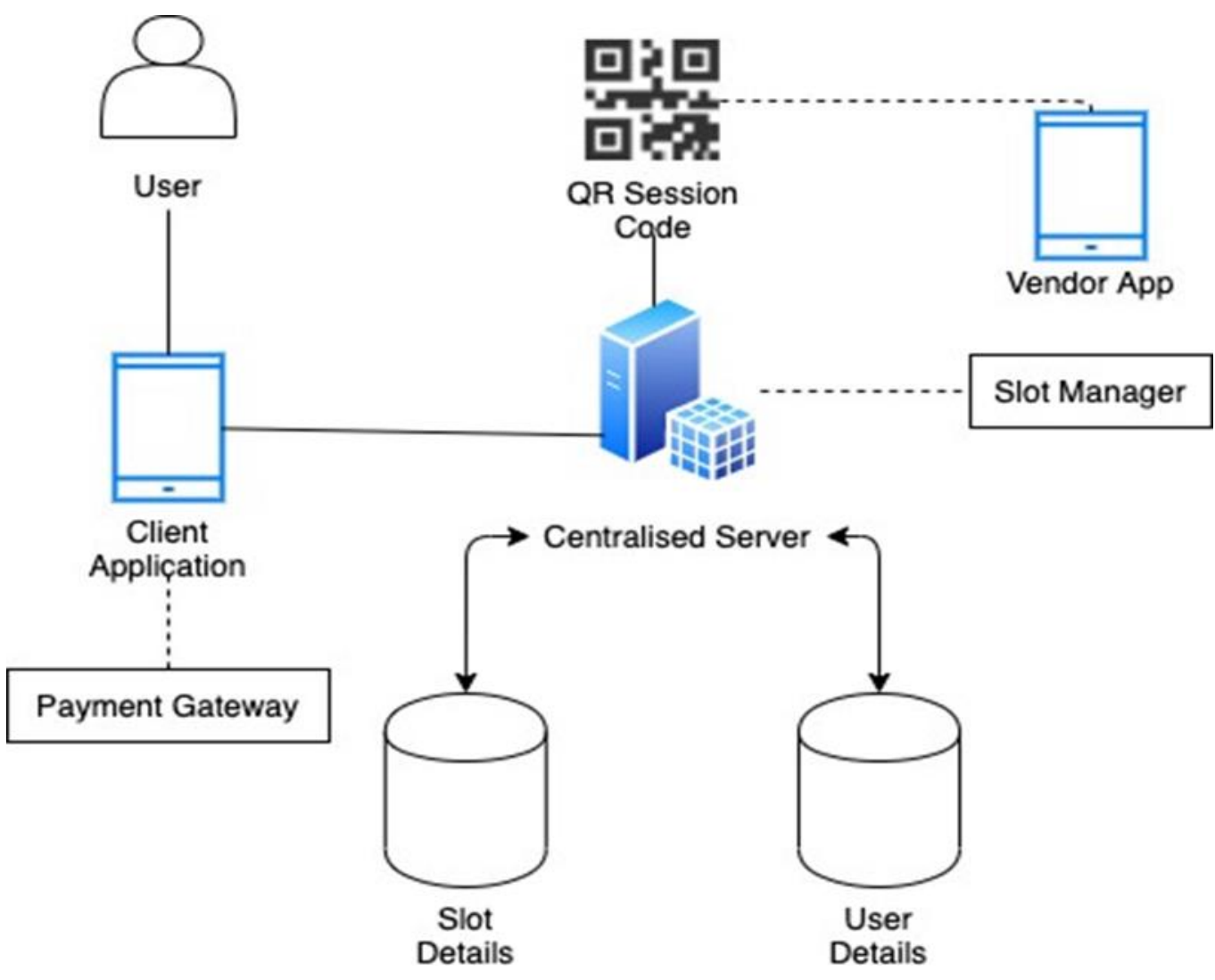

Figure 1 Architecture Diagram 
o sendconfirmation

- Find loc_lat, loc_long ( Location co- ordinates)

- Find number of parking lots near the location

- Extract parking lotco-ordinates

- $\quad$ Find the nearest parkinglots

- Extract the status of the parkingspaces

- IF status =available

o Then pin the parkinglots

- IF status = notavailable

o Pin the nearest available parkinglot

Reservation Module

- Read loc_name,start_time

- Extractstatus

- If start_time.status =available

If start_time.status = not available

o Estimate the parking time and queue theuser

- Read loc_name,user_status

- If user_status =reserved,

o Send confirmationmessage

- IF user_status =queued,

o Send the user a queueing message

o Update waittime

o Allot when slotavailable

Output: The output is a prompt on the client application screen.

\section{Conclusion}

The proposed system helps the user find available parking spaces and reserve them for their use when they reach their desired destination. The estimation algorithm helps the systeminallowingtheuserstobooktheslotsas per their liking without any disturbance. This system can be implemented to efficiently manage the parking spaces and also make the paymenteasy. Below we have attached the graphs for the required and available parking slots both on weekdays and weekends. With our system we can balance this gap in an efficient manner.

\section{References}

1. Jiazao Lin, Shi-Yong Chen, Chih-Yung Chang, Guilin Chen, "SPA: Smart Parking Algorithm Based on Driver Behavior and Parking Traffic Predictions",2019,

2. Volume: 7, Journal Article, IEEE Access

3. Javier Martinez Garcia, Dominik Zoeke,Martin Vossiek, "MIMO-FMCW Radar-Based Parking Monitoring Application With a Modified Convolutional Neural Network With Spatial Priors", 2018, Volume: 6, Journal Article, IEEEAccess

4. Hongwei Wang ; Wenbo He, "A Reservation-based Smart Parking System”, 2011 IEEE Conference on Computer Communications Workshops (INFOCOM WKSHPS), 2011, Conference Paper,Publisher:IEEE

5. Yongmin Zhang, Lin Cai, "Dynamic Charging Scheduling for EV Parking Lots With Photovoltaic Power System",2018,

6. Volume 6, Journal Article, IEEE Access

7. Maigha, M. L. Crow, “A Transactive OperatingModelforSmartAirportParking Lots ”, IEEE Power and Energy Technology Systems Journal, 2018, Volume: 5, Issue: 4, Journal Article,IEEE

8. Zhang Zhaoyun, Zhao Wenjun, Yang Mei, Kang Li, Zhang Zhi, Zhao Yang, Liu Guozhong,YaoNa, “Applicationofmicro- grid control system in smart park”, The

9. Journal of Engineering, 2019, Volume: 2019, Issue: 16, Journal Article, IET

10. Faan Hei Hung, Kim-Fung Tsang, Chung Kit Wu, Yang Wei, Yucheng, Wang Hao, "Cost and Time-Integrated Road-to-Park Cruising Prevention Scheme in Smart Transportation”,IEEE Access, 2019,

11. Volume: 7, Journal Article, IEEE 
12. Heng Li, Yonghe Liu, Zheng Qin, Huigui Rong, Qin Liu, “A Large-Scale Urban Vehicular Network Framework for IoT in Smart Cities", IEEE Access Year: 2019|Volume: 7 |Journal Article | Publisher:IEEE

13. Hansoo Kim, Sungjin Cho, ChulhoonJang, Myoungho Sunwoo, Kichun Jo, "Evidence Filter of Semantic Segmented Image From Around View Monitor in Automated Parking System", IEEE Access, 2019,

14. Volume: 7, Journal Article, IEEE

15. Kamran Sattar Awaisi, Assad Abbas, Mahdi Zareei, Hasan Ali Khattak, Muhammad Usman Shahid Khan, Mazhar Ali, Ikram Ud Din, Sajid Shah, "Towardsa Fog Enabled Efficient Car Parking Architecture", IEEE Access, 2019, Volume: 7, Journal Article,IEEE.

16. Sampathkumar, A., Murugan, S., Sivaram, M., Sharma, V., Venkatachalam, K. and Kalimuthu, M., 2020. Advanced Energy Management System for Smart City Application Using the IoT. In Internet of Things in Smart Technologies for Sustainable Urban Development (pp. 185-194). Springer, Cham.

17. Sampathkumar, A., Murugan, S., Rastogi, R., Mishra, M.K., Malathy, S. and Manikandan, R., 2020. Energy Efficient ACPI and JEHDO Mechanism for IoT Device Energy Management in Healthcare. In Internet of Things in Smart Technologies for Sustainable Urban Development (pp. 131-140). Springer, Cham. 